\title{
Ciclo e indicadores antecedentes na indústria do Rio Grande do Sul
}

Igor Alexandre Clemente de Morais Professor do Programa de Pós-Graduaçáo em Economia da Unisinos

\author{
Palavras-chave \\ ciclos dos negócios, quebras \\ estruturais, indicadores \\ antecedentes.
}

Classificação JEL C32, C50, E32

\section{Key-Words}

business cycles, structural breaks, leading indicator.

JEL Classification C32, C50, E32

\section{Resumo}

Esse artigo tem dois principais objetivos. Primeiro compara três diferentes técnicas de datação de ciclos para determinar os picos e os vales da produção industrial do Rio Grande do Sul. Os resultados apontaram que, entre 1991-I e 2008-IV, o setor no Estado passou por cinco recessóes. Duas tiveram curta duração, de quatro trimestres, e estáo associadas a uma taxa de câmbio mais valorizada. Dois outros ciclos recessivos ocorreram em uma conjuntura de crises internacionais. Por fim, a mais longa recessão, que durou oito trimestres, teve como vetor a estiagem que atingiu o Estado. Um segundo objetivo é a determinação de indicadores antecedentes da produção industrial gaúcha. Partindo de mais de 200 séries candidatas, encontra-se que quatro conseguem antecipar a dinâmica cíclica do setor.

\section{Albstract}

This article has two principal goals. First, it compares three different techniques for dating cycles to determine peaks and through of industrial production in the state of Rio Grande do Sul. The results show that between 1991-I and 2008-IV, this sector of the economy underwent five recessions. Two of these were short, lasting four quarters, and are associated with a higher exchange rate. Two other recessives cycles occurred in scenarios of international crises. Finally, the longest recession, lasting eight quarters, was related to the drought that affected the State in 2005. A second objective is the determination of leading indicators of industrial production. Starting with more than 200 series, we found that four are associated with cyclical periods. 


\section{1_Introdução}

Alguns pontos importantes emergiram do cenário de crise internacional, ao final da década de 2000, como a discussão sobre ciclos dos negócios e a literatura que trata da mensuração de indicadores coincidentes, antecedentes e defasados, segundo Kose et al. (2008), que analisam os movimentos cíclicos globais.

Há anos que os economistas se preocupam com a análise dos ciclos. Os primeiros estudos remontam ao século XIX, sendo comum uma relação do ciclo com eventos climáticos, ou seja, o comportamento da produção de grãos e seus preços praticados no mercado, $\operatorname{com}^{1}$ as "manchas solares". Além disso, as pesquisas debruçaram-se mais na conceituação e na caracterizaçáo dos ciclos e pouco com o desenvolvimento de um método estatístico minimamente eficiente e dinâmico. $\mathrm{O}$ artigo seminal de Burns e Mitchell (1946) e posteriormente os trabalhos de Bry e Boschan (1971) e Stock e Watson (1988, 1989, 1991 e 1993) inauguraram uma nova etapa na discussão sobre esses fenômenos na ciência econômica, incorporando o uso mais rigoroso da estatística.

Tradicionalmente, os ciclos são divididos em duas fases: a expansão e a contração. Esta análise, porém, pode ser completada com os movimentos que ocorrem entre elas. Na literatura da área, adota-se que, quando há o término de um período de expansão, caracterizado como a formação de um pico, se tem início uma recessão. Quando se forma um vale, indicando que o período de contração se encerrou, inicia-se a expansão. Ou seja, uma contração não necessariamente representa uma queda absoluta na atividade econômica, podendo estar relacionada ao declínio da taxa de crescimento. Note que essa definição é diferente da clássica, que assume a recessão como uma sequência de quedas na atividade. Burns e Mitchell (1946) consideram a possibilidade de existência de nove estágios do ciclo, avaliando-os a partir do TPT (trough-peak-trough) e do PTP (peak-trough-peak).

Nessa linha, a análise do ciclo dos negócios é feita valendo-se de dois conjuntos de abordagem: o ciclo específico e o ciclo de referência. No primeiro caso, compara-se a mudança cíclica de uma atividade econômica com uma estrutura cronológica dos picos e dos vales da própria atividade em questão. Assim, a duração da amplitude ${ }^{2}$ de uma queda específica é

$\begin{array}{ll}{ }_{1}^{1} \text { Alguns autores chegavam até } & \text { dois pontos. Por exemplo, } \\ \text { a associar o ciclo econômico } & \text { uma amplitude de queda } \\ \text { aos movimentos dos planetas. } & \text { é dada pela diferença de } \\ \text { Uma discussáo interessante } & \text { tempo entre o pico e o vale. } \\ \text { sobre esse assunto pode ser } & \text { Uma amplitude de um ciclo } \\ \text { vista em Morgan (1990). } & \text { de crescimento é dada pela } \\ { }^{2} \text { A amplitude de um ciclo } & \text { diferença entre o vale e o pico } \\ \text { é dada pela diferença entre } & \text { desse ciclo. }\end{array}$


medida entre um pico e o vale. Portanto, quedas da atividade industrial, por exemplo, são confrontadas com os períodos cíclicos do setor industrial. De outra forma, pode-se comparar essa queda com a estrutura cronológica de uma atividade de referência, que pode ser a economia como um todo. Nesse caso, tem-se a análise de ciclo de referência. ${ }^{3}$

Do ponto de vista da economia como um todo, náo basta olhar apenas um indicador para definir um momento recessivo. A indústria pode estar em recessão, mas a agropecuária e o setor de serviços apresentarem ciclos diferentes. Da mesma forma, o emprego pode estar se retraindo, todavia as vendas do comércio e a renda continuam a se expandir por determinado período. Assim, a caracterização de uma recessão deve contemplar não apenas o comportamento de uma única variável, mas um movimento conjunto de indicadores representativos da atividade econômica. ${ }^{4}$

${ }^{3}$ Bry e Boschan (1971) sugerem um algoritmo para encontrar os picos e os vales de uma série de dados. Hamilton (1989, 1990, 1991 e 1996) propôs o uso dos modelos de mudança de regime markoviano para captar esses movimentos, considerando diversas outras características dos ciclos.
Há uma série de estudos que procuram verificar os movimentos cíclicos de séries de dados de um país, como pode ser observado em Picchetti e Toledo (2002), Morais e Frainer (2005), Issler e Vahid (2006), Céspedes et al. (2006), Morais e Portugal (2007), ou então, entre diferentes países, como se pode ver em Engle e Issler (1993), Artis e Zhang (1997), Gregory et al. (1997), Kose et al. (2003), Stock e Watson (2005), Canova et al. (2005) e Kose et al. (2008).

A praticidade e a importância desses resultados para ajudar na interpretação do cenário econômico impulsionaram a pesquisa na área. Nos EUA, uma vez que o NBER (National Bureau of Economic Research) determina os picos e os vales de um ciclo, tais datas passam a servir de referência na literatura. Para uma revisão e aplicação desses conceitos, ver Stock e Watson (1993) e Zarnowitz (1996). A análise de ciclo também é implementada por outros organismos internacionais. A OCDE calcula um conjunto de indicadores antecedentes para várias economias - ver OECD (2008), para uma discussão da metodologia, Seip e McNown (2007) e Nilsson e Guidetti (2008), para uma avaliação da performance dos indicadores antecedentes diante da análise dos ciclos.

Ao final da década de 2000, foi criado no Brasil o Comitê de Datação de 
Ciclos Econômicos (CODACE), que se propóe a fazer a datação dos picos e dos vales da economia, mas sem se aprofundar na discussão sobre indicadores antecedentes, coincidentes e defasados (IBRE, 2009).

Como forma de contribuir para a discussáo e a aplicação dessa literatura no Brasil, este artigo tem dois principais objetivos. Em primeiro lugar, abordar as características cíclicas da produção industrial no Rio Grande do Sul em uma perspectiva histórica. Isso vai ajudar a entender alguns fatos estilizados, como a duração dos ciclos, a intensidade deles e as variáveis a ser analisadas e que antecipam seus movimentos. $\mathrm{O}$ segundo objetivo é avaliar o conjunto de indicadores econômicos que podem ser definidos como antecedentes da produção industrial gaúcha. Destaca-se que o conhecimento desses permitirá criar uma ferramenta estatística de antecipação dos movimentos cíclicos do setor no Estado. Até o momento, esse é o primeiro exercício de identificação nessa linha de pesquisa para dados setoriais em âmbito regional.

Para tanto, este artigo está dividido em quatro seções, além desta introdução. $\mathrm{Na}$ segunda seção, é ilustrada a metodologia empregada na datação dos picos e dos vales. Na seção três, é feita a descrição dos dados e discutidos os resultados dos ciclos. $\mathrm{Na}$ seção quatro, são realizadas as estimativas dos indicadores antecedentes. E, por fim, na seção cinco, estão as principais conclusóes.

\section{2_Metodologia}

Há diversos métodos, paramétricos e não paramétricos propostos na literatura internacional para se datar o ciclo dos negócios (Harding; Pagan, 2005). Neste artigo, são usadas três técnicas, duas não paramétricas e uma paramétrica.

Uma "regra de bolso" útil para definir recessão em uma economia é identificar duas quedas consecutivas ${ }^{5}$ do PIB real, ou entáo outros indicadores, como a indústria. Alternativamente, pode-se usar a proposta de Bry e Boschan (1971), doravante denominada de "B-B". Destacase que ambas as técnicas são consideradas não paramétricas. Por fim, este artigo também aplica a proposta paramétrica de Hamilton (1989).

A técnica de B-B usa algumas regras específicas, como, por exemplo: (i) um limite mínimo de 15 meses para os ciclos; (ii) a duração de uma expansão ou de uma contração não pode ser menor que cinco meses e; (iii) nenhum turning point é considerado em um intervalo de seis meses, antes do fim da série, ou depois de seu início. $O$ processo de datação nessa metodologia contempla a elimina-

\footnotetext{
${ }^{5}$ Essa pode ser considerada muito mais indicativa do que determinística para caracterizar uma recessão.
} 
ção de valores mínimos e máximos que são extremos e o uso de médias móveis em duração de 3 a 6 meses, dependendo da dominância cíclica mensal.

A proposta de Hamilton (1989) mostra-se mais robusta, simples e transparente. Para uma aplicação e discussão dos resultados comparativamente ao método não paramétrico, ver Harding e $\mathrm{Pa}$ gan (2003).

De forma geral, partindo de um modelo de regressão linear sem mudança de regime, $y_{t}=x_{t} \beta+\varepsilon_{t}, \operatorname{com} \varepsilon_{t}-\operatorname{NID}\left(0, \sigma^{2}\right)$ os parâmetros $\beta$ e $\sigma^{2}$ podem ser estimados a partir da maximização do $\log$ da função de verossimilhança $L n(L)=\sum_{t=1}^{T} \ln \left[f\left(y_{t}\right)\right]$, onde $f($.) é função de densidade.

Entretanto, se há quebra estrutural nos parâmetros, temos $y_{t}=x_{t} \beta_{s_{t}}+\varepsilon_{t}$, em que $\varepsilon_{t}-\operatorname{NID}\left(0, \sigma_{s_{t}}^{2}\right)$, com $s_{t}=1,2, \ldots n$. Neste caso, a funçáo $\log$ da verossimilhança passa a ser dada por $\operatorname{Ln}(L)=\sum_{t=1}^{T} \ln \left[f\left(y_{t} / s_{t}\right)\right]$, e os parâmetros a ser estimados são $\left(\beta_{0}, \beta_{1}, \ldots, \beta_{n}, \sigma_{1}, \sigma_{2}, \ldots \sigma_{n}\right)$.

Quando se conhecem os momentos em que ocorreram as quebras estruturais e o número de "regimes" que vai caracterizar a série de dados, a estimativa dos parâmetros é mais simples. Contudo, se $s_{t}$ é desconhecido, então o processo de estimação é mais complexo (Hansen, 1992; Hamilton, 1996).

Para estimar essa função de densidade, é necessário fazer uma hipótese sobre o comportamento estocástico de $s_{t}$ que pode ser tanto dependente quanto independente de seus valores passados. $\mathrm{O}$ caso mais interessante, porém, é quando o regime no momento $t$ depende do regime nos momentos anteriores. Esse processo é denominado de "mudança markoviana de ordem $k$ ". Assim, como podem existir $k$ estados, as probabilidades de transição entre esses estados podem ser representadas por meio de uma matriz de probabilidade de transição $P=\left[p_{i j}\right] \in M(k x k)$ tal como:

$$
P=\left[\begin{array}{cccc}
p_{11} & p_{21} & . . & p_{k 1} \\
p_{12} & p_{22} & . . & p_{k 2} \\
: & : & : & : \\
p_{1 k} & p_{2 k} & . . & p_{k k}
\end{array}\right]
$$

onde:

$\sum_{j=1}^{k} p_{i j}=1$ para $i=1,2, \ldots, k, p_{i j} \geq 0$ para $i, j=1,2, \ldots \ldots, k$, e o vetor de probabilidade de transição de Markov é dado por $P=\left(P_{11}, \ldots P_{k k}\right)^{\prime},\left(k^{2} \times 1\right)$. Esses resultados 
permitem que seja possível determinar o tempo de duraçáo que se permanece em cada estado.

O processo de estimação é feito por máxima verossimilhança e, para que sejam estimados os parâmetros do modelo $\theta=\left(\mu_{1}, \mu_{2}, \ldots, \mu_{k}, \sigma_{1}^{2}, \sigma_{2}^{2}, \ldots, \sigma_{k}^{2}\right)$, é necessário fornecer os valores iniciais $\theta^{(0)}$. Um algoritmo ${ }^{6}$ conhecido como EM (Expectation-Maximization) foi proposto por Hamilton (1990) e também está descrito em Ruud (1991).

Duas dificuldades aparecem no processo de estimação: (i) o uso de modelos mais complexos, com muitas variáveis, que dificulta a maximização da verossimilhança; (ii) a determinação do número de estados ou de regimes, especialmente porque se está tentando fazer um teste de hipótese em modelos em que o parâmetro de distúrbio não é identificado sob a hipótese nula. Desta forma, a distribuição assintótica do teste de razão de verossimilhança não é padrão. Alguns autores propuseram diversos testes para verificar a não linearidade com uma distribuição nula assintótica (Hansen, 1992; Garcia, 1998). Para verificar a adaptação destes modelos aos dados, podem ser utilizados os testes de especificação propostos por Engel e Hamilton (1990) e Hamilton (1996).

\section{3_Ciclos na indústria do Rio Grande do Sul}

A indústria do Rio Grande do Sul, apesar de diversificada ${ }^{7}$ em segmentos, produtos e mercados, ${ }^{8}$ tem forte presença das categorias de bens de capital e de consumo durável, ambos muito dependentes da renda, do volume de crédito e da taxa de juros. Além disso, boa parte das vendas setoriais tem orientação exportadora, como é o caso de calçados, fumo e alimentos e bebidas. Talvez exatamente pela presença desses dois vetores que o setor industrial regional experimenta, de forma mais contundente, os impactos de juros maiores, valorização cambial ou a queda da atividade econômica externa.

A partir da "regra de bolso" não paramétrica de duas quedas consecutivas do produto e analisando os dados trimestrais ${ }^{9} \mathrm{da}$ produçáo industrial do Rio Grande do Sul,
${ }^{9}$ Os dados mensais são do IBGE, Tabela 2295. Foi usado o filtro X-12 para dessazonalizar cada seção de atividade industrial e depois esses foram trimestralizados. Apesar de a aplicação de filtros como esse modificar algumas características dos dados, a escolha pela dessazonalização tem como objetivo reduzir a incidência desses padróes nos resultados dos ciclos. Esse ponto é discutido na seção 4 .


${ }^{10}$ Agradeço a Mark Watson por disponibilizar a rotina do GAUSS para implementar a metodologia de B-B. Todos os resultados estatísticos desta e da próxima seção foram omitidos por economia de espaço e podem ser enviados mediante solicitação.

\section{Gráfico 3.1_Ciclos recessivos na indústria do RS (regra de bolso)}

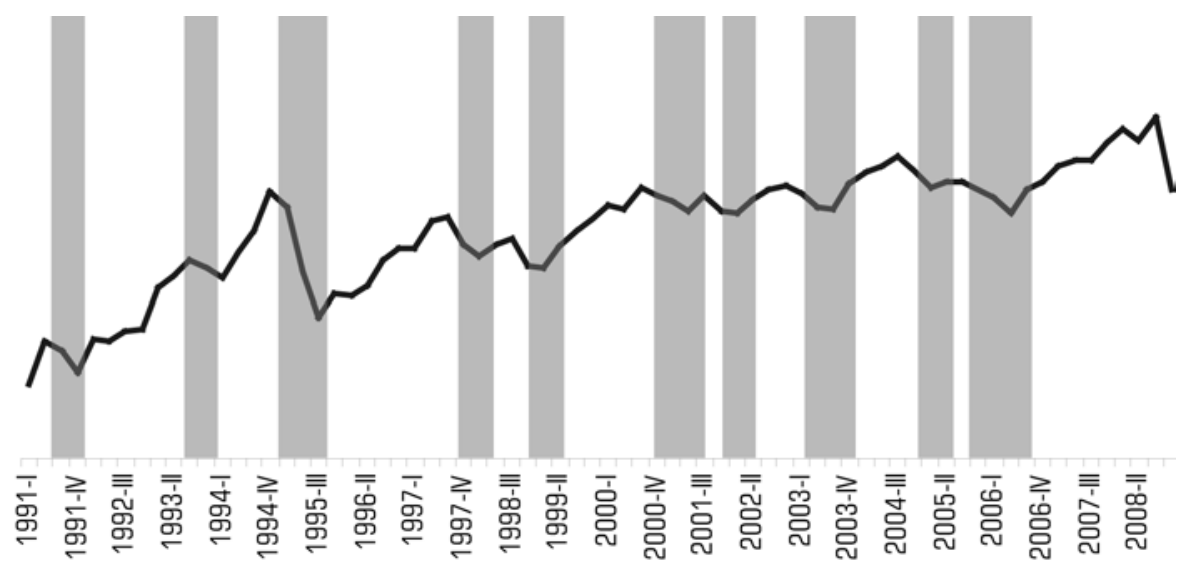

Fonte de dados brutos: IBGE. Critério de seleçâo das recessôes, dois trimestres de queda.

entre 1991-I e 2008-IV, podemos ver que há indícios de dez recessões nesse período, mostradas nas barras cinza no Gráfico 3.1. A mais longa recessão estaria relacionada ao período da estiagem que atingiu o Estado no ano de 2005, que, somada à forte valorização cambial, prejudicou sobremaneira a atividade industrial. Para esse período em questão, a duração estimada da recessão foi de quatro trimestres, com início em 2005-III e se estendendo até 2006-II.

Apesar de útil para ilustrar os períodos cíclicos da economia, essa "regra de bolso" pode falhar em captar de maneira fidedigna esses movimentos. Ou seja, podemos ter dois trimestres consecutivos de queda que seja de pequena magnitude, a ponto de náo representar a consolidaçáo de um período recessivo. Da mesma maneira, a redução na taxa de crescimento pode estar associada ao fim do pico, indicando o início de um período recessivo e que não é captado por essa definição clássica, isto é, os picos e os vales podem se manifestar em um período de tempo anterior ao indicado pela "regra de bolso".

Usando a proposta de Bry e Boschan (1971) para dados mensais, tem-se uma quantidade menor de ciclos recessivos. Nesse caso, são cinco períodos de recessão, ou seja, o intervalo de tempo em que a atividade produtiva sai de um pico de produção para atingir o vale. Esses estão sinalizados no Gráfico 3.2, a partir das barras cinza. ${ }^{10}$

O menor ciclo ocorreu no início do Plano Real, com duração de nove me- 
ses, entre dezembro de 1994 e setembro de 1995. O fato é que, mesmo diante da estabilidade de preços, a valorização do real em muito prejudicou as exportaçóes industriais gaúchas, com impactos significativos na produção.

Dois outros ciclos chamam a atenção pela alta duração. $\mathrm{O}$ primeiro, com início em abril de 1997, estendeu-se até janeiro de 1999, perfazendo um total de 21 meses. Nesse período, a valorização do real, bem como a menor atividade econômica mundial na esteira das crises financeiras da Ásia e da Rússia, é um dos dois principais fatores a explicar a retração na produção industrial gaúcha, muito ligada ao setor externo.

O segundo ciclo recessivo de grande duração ocorreu entre julho de $2004 \mathrm{e}$ abril de 2006. Entre os motivos apontados pelo fraco desempenho da indústria local, destaque para os impactos da estiagem, principalmente atingindo a cultura da soja, seguida da valorização da taxa de câmbio. Ambas as variáveis deprimiram a receita dos produtores ligados ao agronegócio e prejudicaram os exportadores industriais. Para uma discussão sobre a conjuntura econômica regional desse período, ver Morais (2006).

Como forma de confrontar os resultados não paramétricos anteriores, utiliza-se a proposta paramétrica ${ }^{11}$ de Hamil-

\section{Gráfico 3.2_Ciclos recessivos na indústria do RS (método de Bry-Boschan)}

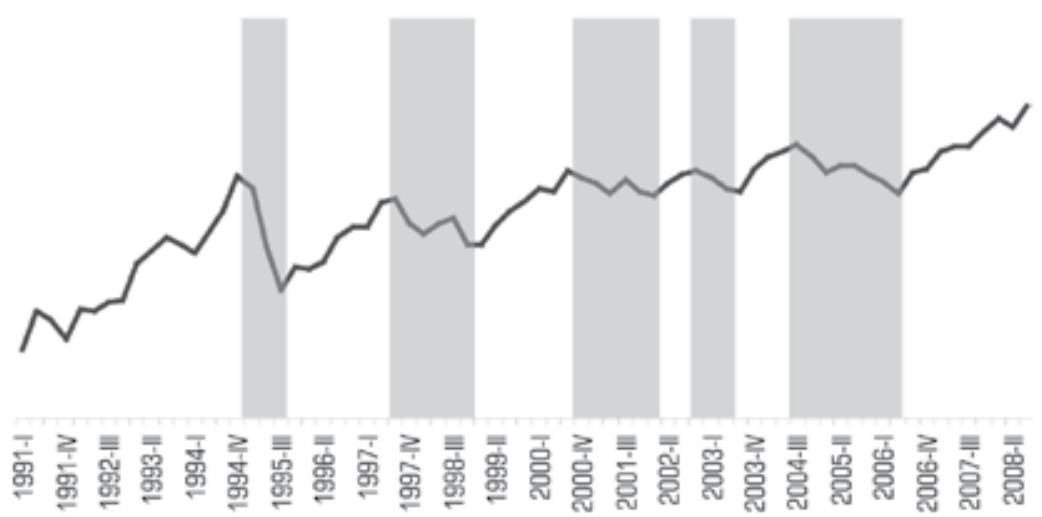

Fonte de dados brutos: IBGE. Critério de seleção das recessōes, Bry-Boschan (1971).

ton $(1989,1990,1991,1996)$ para dados trimestrais. Nesse caso, são encontrados 11 períodos que podem ser classificados como recessivos. ${ }^{12}$ Contudo, desses, cinco foram de curta duração, com apenas um trimestre e, mesmo sendo mostrados pelas barras cinza, no Gráfico 3.3, não foram classificados como recessão.

Pode-se ver que, no período compreendido entre 1996-I e 2008-IV, a indús-

$\begin{array}{ll}{ }^{11} \text { De formulaçáo estatística } & \text { ou de recessão, sem que, para } \\ \text { mais complexa, o modelo } & \text { tanto, se perca a informação } \\ \text { assume a existência de } & \text { de intensidade e assimetria. } \\ \begin{array}{l}\text { uma variável de estado } \\ \text { estatisticamente não }\end{array} & \begin{array}{l}{ }^{2} \text { Dada a excessiva } \\ \text { volatilidade dos dados no } \\ \text { observável que vai determinar }\end{array} \\ \begin{array}{l}\text { se a economiano encontra-se em } \\ \text { um regime de crescimento }\end{array} & \begin{array}{l}\text { tiveram início no primeiro } \\ \text { trimestre de } 1996 .\end{array}\end{array}$


Gráfico 3.3_Ciclos recessivos na indústria do RS (método de Hamilton)

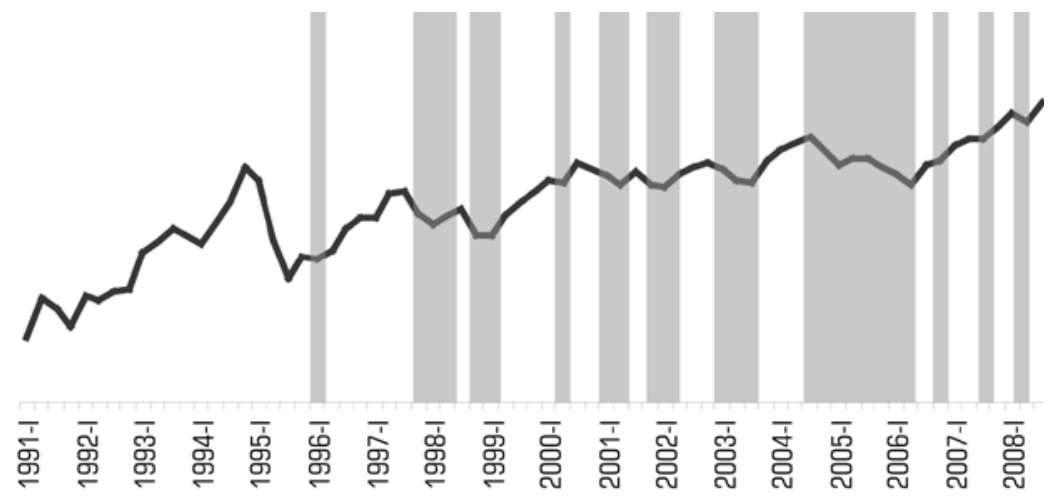

Fonte de dados brutos: IBGE. Critério de seleçāo das recessões, Hamilton (1989) - MSM(2)-AR(4). tria do Rio Grande do Sul atravessou seis períodos recessivos, e o mais longo pode ser associado ao mesmo cenário de adversidades indicado anteriormente pela metodologia de B-B. Após atingir um pico de produção em 2004-II, a indústria iniciou um período de retração que perdurou até 2006-II, completando oito trimestres entre o pico e o vale.

Outros dois momentos históricos podem ser caracterizados como recessivos. O primeiro, entre 1997-III e 1998-II, coincide com as indicaçóes das estimativas anteriores via B-B. Sob um cenário de crise financeira na Ásia e, posteriormente, na Rússia, além da valorização da taxa de câmbio, a indústria completou um ciclo de quatro trimestres de recessão. A seguir, após momentos de curta retração, um novo ciclo longo tem início em 2002IV, também durando quatro trimestres e se encerrando em 2003-III.

Além das datas referentes aos ciclos de expansão e recessão, outros resultados interessantes surgem com base no método paramétrico. Em primeiro lugar, a taxa média de crescimento da produção industrial para cada estado da economia. Nesse caso, quando essa se encontrava em períodos recessivos, a produção setorial caiu a uma taxa média de $1,36 \%$ ao trimestre. Por outro lado, em momentos de expansão, saindo de um vale em direção ao pico, a taxa média de crescimento foi da ordem de $3,2 \%$ ao trimestre. ${ }^{13}$

O segundo resultado interessante diz respeito à duração. As estimativas indicam que, em média, uma recessão na indústria do Rio Grande do Sul dura cerca de 2,6 trimestres, ao passo que uma expansão, 2,1 trimestres. Essas são estimativas para uma média, ocorrendo ciclos de mais longa duração, como pode ser visto no caso da recessão que teve início em 2004-III e que se estendeu até 2006-II. A Tabela 1 faz um comparativo dos resultados das três técnicas.

Por fim, têm-se as estimativas das probabilidades de se estar em cada um dos regimes cíclicos, indicadas na matriz de probabilidade de transição. Uma vez 
Tabela 1_Estimativa dos ciclos recessivos da indústria do Rio Grande do Sul

\begin{tabular}{|c|c|c|}
\hline Regra de bolso & Bry-Boschan (1971) & Hamilton (1989) \\
\hline \multicolumn{3}{|l|}{ 91-III a 91-IV } \\
\hline \multicolumn{3}{|l|}{ 93-IV a 94-I } \\
\hline \multirow[t]{2}{*}{ 95-I a 95-III } & 94-IV a 95-III & \\
\hline & & 96-I a 96-I \\
\hline 97-IV a 98-I & & 97-III a 98-II \\
\hline \multirow[t]{2}{*}{ 98-IV a 99-I } & 97-II a 99-I & 98-IV a 99-I \\
\hline & & 00-II a 00 -II \\
\hline $00-\mathrm{IV}$ a $01-\mathrm{II}$ & \multirow[b]{2}{*}{ 00-III a $02-\mathrm{I}$} & 00-IV a 01-II \\
\hline $01-I V$ a $02-I$ & & 01-IV a 02-I \\
\hline 03-I a 03-III & 02-III a 03-III & 02-IV a 03-III \\
\hline \multicolumn{3}{|l|}{ 04-IV a $05-\mathrm{I}$} \\
\hline \multirow[t]{4}{*}{ 05-III a 06-II } & 04-II a 06-II & 04-III a 06-II \\
\hline & & 06-IV a 06-IV \\
\hline & & 07-III a 07-III \\
\hline & & 08-II a 08 -II \\
\hline \multicolumn{3}{|c|}{ Média de duração dos ciclos recessivos (em trimestres) } \\
\hline 2,5 & 5,4 & 2,6 \\
\hline
\end{tabular}

atingido o período recessivo, há uma probabilidade de $38 \%$ de se passar para um cenário de crescimento no trimestre seguinte. Por outro lado, estando em um ciclo de crescimento, há probabilidade média de $47 \%$ de se entrar em um período recessivo no trimestre posterior. ${ }^{14}$ Nota-se que há assimetria na transição entre o ciclo de expansão e o de recessão, com chance maior de se entrar nos períodos recessivos.

Nesse ponto, podemos tirar algumas conclusões sobre a classificação dos ciclos na indústria do Rio Grande do Sul. O primeiro é que, mesmo com a utilização de três técnicas distintas, é possível caracterizar as datas em que o setor realmente estava em recessão. O segundo ponto é a duração. Desconsiderando os ciclos recessivos de curta duraçáa, como os de um trimestre, e preservando apenas aqueles que apresentam similaridade entre os três métodos anteriormente utilizados, podemos observar que, no período pós-Plano Real, a indústria do Rio Grande do Sul
${ }^{14}$ Esses resultados referen-se a médias para todo o período, ocorrendo momentos em que a probabilidade de se estar em um período de crescimento e entrar em recessão é maior ou menor que o especificado. 
Tabela 2_Ciclos recessivos da indústria do Rio Grande do Sul

\begin{tabular}{|c|c|c|c|c|c|c|}
\hline & \multicolumn{2}{|l|}{ Esse artigo } & \multicolumn{2}{|c|}{ Chauvet e Morais (2008) } & \multicolumn{2}{|l|}{ IBRE (2009) } \\
\hline & Pico & Vale & Pico & Vale & Pico & Vale \\
\hline Ciclo 1 & 1994-IV & 1995-III & 1995-II & 1995-III & 1995-I & 1995-III \\
\hline Ciclo 2 & 1997-III & 1999-I & 1998-III & 1998-IV & 1997-IV & 1999-I \\
\hline Ciclo 3 & 2000-IV & 2002-I & 2001-II & 2001-IV & 2001-I & 2001-IV \\
\hline Ciclo 4 & 2002-IV & 2003-III & 2003-I & 2003-II & 2002-IV & 2003-II \\
\hline Ciclo 5 & $2004-\mathrm{III}$ & 2006-II & & & 2008-III & \\
\hline
\end{tabular}

Nota: *A última reuniấo do CODACE para definir os ciclos recessivos no Brasil ocorreu em 27 de maio de 2009.

experimentou cinco ciclos recessivos. Esses resultados são mostrados na Tabela 2.

O ciclo 1, com início nos primeiros meses do Plano Real, vai de 1994-IV a 1995-III, com duração de quatro trimestres. O segundo ciclo teria duração de sete trimestres, estendendo-se de 1997-III a 1999-I. Dois pontos merecem destaque. Primeiro é a existência de um fator externo comum, a taxa de câmbio valorizada e, segundo, o fato de os dois ciclos serem similares ao movimento encontrado para o PIB do Brasil. Em ambos, nota-se queda conjunta da produção industrial nacional e gaúcha e também de diversos indicadores de atividade do setor no Estado, como as vendas, o emprego e as horas trabalhadas na indústria (Tabela 3). Destaca-se que a duração maior do segundo ciclo recessivo pode estar associada à presença de várias crises no cenário internacional, como a da Ásia e a da Rússia, e a consequente desvalorização do real a partir do primeiro trimestre de 1999.

A seguir, após um período de crescimento da atividade industrial, o terceiro ciclo de recessão se iniciaria em 2000IV, estendendo-se até 2002-I e perfazendo seis trimestres. Esse intervalo engloba a crise da Argentina, o estouro da bolha do mercado acionário norte-americano, os problemas de contabilidade nas empresas americanas e os atentados terroristas nos EUA. Todavia, antecede o ciclo recessivo identificado para o Brasil em cerca de dois trimestres. Como a estimativa de recessão não precisa estar necessariamente associada a variaçóes negativas na série em questáo, os resultados podem estar refletindo a forte desaceleração da indústria do Rio Grande do Sul, que ocorreu a partir de setembro de 2000.

De fato, nesse período, a produção industrial do Estado tem a menor queda 
Tabela 3_Comportamento de algumas variáveis macroeconômicas nos ciclos recessivos (var. \% média ao trimestre)

\begin{tabular}{|c|c|c|c|c|c|}
\hline & Ciclo 1 & Ciclo 2 & Ciclo 3 & Ciclo 4 & Ciclo 5 \\
\hline \multicolumn{6}{|l|}{ Brasil } \\
\hline PIB-total & $0.46 \%$ & $-0.09 \%$ & $0.28 \%$ & $0.19 \%$ & $0.64 \%$ \\
\hline Produção Industrial & $-0.93 \%$ & $-0.93 \%$ & $0.28 \%$ & $-0.07 \%$ & $0.82 \%$ \\
\hline Vendas do comércio & & & $-0.25 \%$ & $-1.10 \%$ & $1.21 \%$ \\
\hline Emprego formal & $-0.04 \%$ & $-0.50 \%$ & $0.70 \%$ & $0.68 \%$ & $1.35 \%$ \\
\hline \multicolumn{6}{|l|}{ Rio Grande do Sul } \\
\hline Exportaçóes & & $-4.60 \%$ & $-1.12 \%$ & $2.72 \%$ & $2.06 \%$ \\
\hline Comércio & - & - & $-0.25 \%$ & $-1.01 \%$ & $-0.04 \%$ \\
\hline \multicolumn{6}{|c|}{ Indústria do Rio Grande do Sul } \\
\hline Produção & $-4.77 \%$ & $-1.37 \%$ & $-0.82 \%$ & $-0.92 \%$ & $-1.11 \%$ \\
\hline Vendas & $-6.35 \%$ & $-1.09 \%$ & $-0.19 \%$ & $-4.90 \%$ & $-1.58 \%$ \\
\hline Horas trabalhadas & $-1.07 \%$ & $-1.30 \%$ & $1.63 \%$ & $0.82 \%$ & $-1.50 \%$ \\
\hline IDI* $^{*}$ & $-0.02 \%$ & $0.19 \%$ & $0.54 \%$ & $-0.70 \%$ & $-1.64 \%$ \\
\hline Emprego & $-0.17 \%$ & $-1.22 \%$ & $0.87 \%$ & $0.59 \%$ & $-0.74 \%$ \\
\hline Exportaçóes & & $-4.14 \%$ & $-1.06 \%$ & $2.66 \%$ & $3.07 \%$ \\
\hline
\end{tabular}

Fonte de dados brutos: IBGE, FIERGS, Ministério do Trabalho, SECEX. *Índice de desempenho industrial,calculado com base em uma média ponderada de cinco variáveis do setor industrial.

entre todos os ciclos encontrados (Tabela 3). Além disso, o emprego e as horas trabalhadas permaneceram com crescimento. Esse ciclo recessivo foi longo, porém menos intenso que os demais.

No quarto ciclo, a distância entre o pico de produçáo e o vale seria pequena, de apenas quatro trimestres, com início em 2002-IV e indo até 2003-III. Note que esse período não contempla problemas de valorização da taxa de câmbio nem de cri-

\section{Gráfico 3.4_Produção industrial trimestral - Rio Grande do Sul}

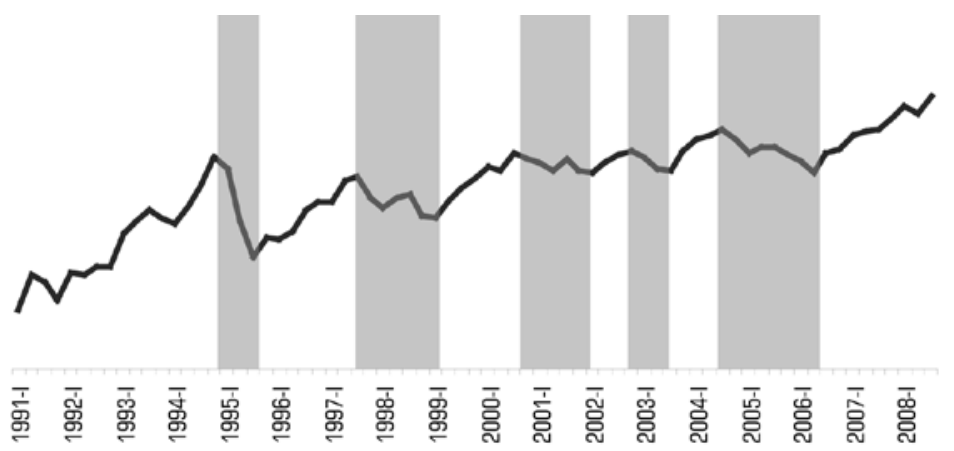

Fonte: IBGE. Critério de seleção das recessôes: mais de um trimestre e similar entre os três métodos. 
ses internacionais, e sim uma crise interna, com as eleições presidenciais de 2002, e a consequente piora nos indicadores macroeconômicos no Brasil. Destaca-se ainda que esse ciclo coincide com as estimativas feitas para o Brasil. Pelos resultados mostrados na Tabela 3 , há coincidência de queda da produção industrial nacional com a gaúcha e também das vendas locais.

Por fim, o último ciclo captado teria duração maior que os demais, isto é, oito trimestres, estendendo-se de 2004-III a 2006-II. Dois importantes motivos podem ajudar a explicar esse longo período recessivo. Em primeiro lugar, o processo de valorização da taxa de câmbio ${ }^{15} \mathrm{e}$, em segundo, um fator local e que pode justificar uma recessão diferente da estimada para o Brasil: a estiagem. As variaçóes apontadas na Tabela 3 evidenciam esse cenário diverso que se alastrou por toda a economia do Estado. O Gráfico 3.4 mostra a evolução do indicador de produção industrial e os

${ }^{15} \mathrm{~A}$ incerteza seria um elemento importante para mudar a decisão dos agentes econômicos. No período aqui considerado, apesar de o Brasil atravessar um cenário político adverso e que poderia ter consequências sobre a volatilidade dos ativos internos, a estimativa de um modelo GARCH $(1,1)$ para a taxa de câmbio mostrou que essa teve pequeno aumento na volatilidade e que não foi suficiente para reverter o forte processo de valorização diante de um cenário internacional favorável para fluxo de capitais. respectivos momentos recessivos, caracterizados pelas barras de cor cinza.

Essa investigação permite inferir a incidência de quatro importantes fatores comuns aos ciclos locais. $\mathrm{O}$ primeiro, em destaque, é uma taxa de câmbio valorizada, presente em três ciclos recessivos, 1, 2 e 5. As diversas crises internacionais seriam outra importante fonte de impacto, verificadas nos ciclos 2 e 3 . Também podemos associar os ciclos a crises internas em razão de problemas macroeconômicos, como nos ciclos 2 e 4, e, por fim, um fator de origem climática como a seca, presente apenas no ciclo 5 .

A classificação dos ciclos em datas, intensidade e duração pode ser complementada com a avaliação dos indicadores antecedentes. $\mathrm{O}$ objetivo principal nos estudos nessa área é determinar quais são os indicadores que podem ser usados para prever, com antecedência, os picos e os vales de diversas variáveis econômicas. No presente caso, usa-se como série de referência a produção industrial e seus ciclos estimados.

Encontrar os indicadores antecedentes da atividade econômica é particularmente importante já que pode auxiliar no processo de decisão por parte de governos, autoridades monetárias, empresas e investidores. Assim, na literatura da área, da mesma forma que cresceu o interesse em 
aperfeiçoar os modelos estatísticos que pudessem dar mais consistência à análise dos movimentos cíclicos, também cresceram os estudos com o objetivo de se antecipar esses movimentos. ${ }^{16}$ Determinar, porém, quando um indicador assume as características coincidentes ou antecedentes de outra variável não é um procedimento simples e requer extenso processo de investigação.

\section{4_Os indicadores antecedentes}

Esta seção tem como objetivo encontrar os indicadores que podem ser considerados como antecedentes da produçáo industrial do Rio Grande do Sul. Para tanto, segue a proposta de Stock e Watson (1989 e 1993), que também foi utilizada por Duarte et al. (2004). O procedimento inicia-se com a seleção de um grande conjunto das mais diferentes variáveis da economia brasileira, relacionadas ao mercado de trabalho, produção industrial por subsetores, consumo, vendas do comércio, de caráter fiscal, do mercado financeiro e de capitais, entre outras. $\mathrm{Na}$ medida do possível, também são utilizados dados regionais.

São empregados dois critérios de seleção de variáveis. O primeiro está relacionado à qualidade dos dados, ou seja, é avaliada a periodicidade que seja su- ficiente para gerar graus de liberdade no estudo de ciclos. Nesse ponto, diversas séries deixam de ser candidatas, uma vez que muitas têm início exclusivamente na década de 1980. Também outras relacionadas à questão fiscal se iniciam apenas no período pós-Plano Real e outras séries, em especial a do mercado de trabalho, foram descontinuadas ao longo do tempo pelos institutos de pesquisa.

Nesse primeiro filtro, de um total de mais de 200 séries, restaram apenas 98, com início em janeiro de 1991 e término em outubro de 2008, perfazendo um total de 214 meses. Essa, porém, ainda é uma quantidade muito elevada de séries. A partir de então, usa-se o segundo critério de seleção, avaliando as que possuem alguma relevância econômica e que podem ter determinada relação com a produção industrial. Tal procedimento nos conduz a um total de 42 séries. Assim, esse seria o conjunto de análise de partida.

Os procedimentos seguintes são utilizados de forma padrão em pesquisas na área de econometria, como a construção de números índice, aplicação do operador log, dessazonalização pelo método $\mathrm{X}-12$ e teste de estacionariedade. Para verificar a existência de raiz unitária sazonal em diferentes frequências, foi usado o procedimento de Beaulieu e Miron (1993), que aplica a proposta de Hylleberg

\footnotetext{
${ }^{16}$ Diversos trabalhos aplicam essa metodologia; ver Stock e Watson (1988, 1989, 1991, 1993) e Chauvet (1998), que usam fatores dinâmicos; Seip e McNown (2007), que usam análise de componente principal; e Estrella e Mishkin (1998) e Chauvet e Morais (2008), que utilizaram um modelo probit.
} 
et al. (1990) para dados mensais. ${ }^{17}$ Os resultados indicam a rejeição da hipótese nula de raiz unitária na frequência zero para todas as séries e ao menos para algumas frequências sazonais, seja com o teste $t$, seja com o $F$ feito em conjunto de duas frequências. Os valores críticos, bem como uma discussão sobre as implicações da raiz unitária sazonal, podem ser vistos em Beaulieu e Miron (1993). Os testes tradicionais de raiz unitária, como ADF e Phillips Perron, apontaram a não estacionariedade das séries em nível. Contudo, na presença de quebra estrutural, esses são viesados. Para identificar essa característica nas séries, aplicaram-se os testes de Andrews-Quandt e Andrews-Ploberger e ambos rejeitam a hipótese nula de ausência de quebra estrutural em to- das as séries. A partir de então, a proposta de Zivot e Andrews (1992) e a de Lee e Strazicich (2004) foram usadas nos testes de Raiz Unitária na presença de quebra estrutural. Os resultados apontaram pela existência de Raiz Unitária em todas as séries, mesmo na presença de quebra estrutural, seja essa no nível, seja então na tendência. ${ }^{18}$

Duas outras técnicas são utilizadas para identificar o tipo de relação que existe entre cada série selecionada e a produção industrial do Rio Grande do Sul. Em primeiro lugar, é feito o teste de causalidade de Granger ${ }^{19}$ com 3, 6 e 12 lags. Nesse caso, de um total de 42 séries, restaram 18 , que possuem algum tipo de evidência de causalidade nas defasagens testadas (ver Anexo 2).
${ }^{17}$ Esse teste foi feito no software RATS 6.0, considerando a presença dos seguintes componentes determinísticos: constante, tendência e dummies sazonais. Como mostrado em Beaulieu e Miron (1993), o polinômio que contém as possíveis raízes unitárias é linearizado em torno de uma raiz unitária na frequência zero. A seguir, o mesmo é estimado a partir dos mínimos quadrados e os valores críticos gerados a partir de uma simulaçáo de Monte Carlo. Neste artigo, os testes de raiz unitária, quebra estrutural, causalidade e correlação cruzada são feitos nas séries com e sem ajuste sazonal. Por fim, a estimativa dos modelos de mudança de regime e de Bry-Boschan para identificação dos ciclos é feita com as séries ajustadas sazonalmente.
${ }^{18}$ Para uma discussão do teste de quebra estrutural, ver Andrews e Ploberger (1994). Os resultados, aqui não apresentados por economia de espaço, podem ser enviados mediante solicitação. Foi utilizado o programa estatístico RATS 6.0.

${ }^{19} \mathrm{O}$ teste é feito para variáveis $\mathrm{I}(0)$, sendo feita a primeira diferença naquelas que indicaram a presença de raiz unitária no procedimento anterior. A seguir, na análise de seleção da existência de causalidade, são excluídas as relaçóes de bi-causalidade, bem como aquelas que apontam que a produção industrial do Rio Grande do Sul causa as demais variáveis. Os mesmos testes foram feitos para as séries sem ajuste sazonal, e os resultados em muito diferem, sinalizando a necessidade de se filtrar essa característica dos dados. 
Tabela 4_Séries antecedentes da produção industrial do Rio Grande do Sul

\begin{tabular}{|c|c|c|}
\hline Série & Defasagens & Fonte \\
\hline Produção industrial - metalurgia dos não ferrosos & 6 & IBGE \\
\hline Vendas - ônibus - nacionais - unidade & 6 e 12 & Anfavea \\
\hline Pessoal ocupado - indústria - índice (média $2006=100$ ) - SP & 3 e 6 & Fiesp \\
\hline Taxa de juros - Over / Selic - (\% a.m.) . . . . . . . . . . & 3,6 e 12 & BCB \\
\hline
\end{tabular}

\section{Gráfico 4.1_Metalurgia de não ferrosos}

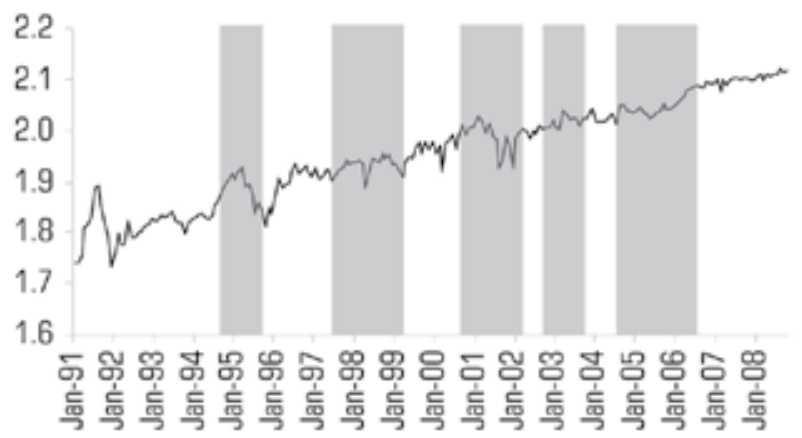

\section{Gráfico 4.2_Vendas de ônibus}

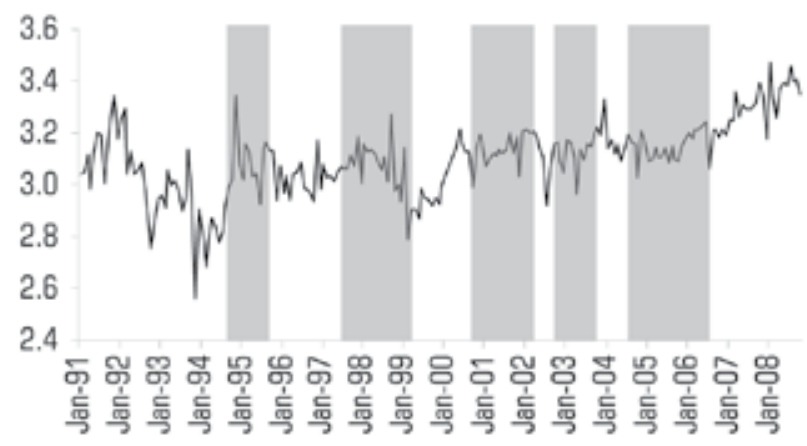

Por fim, para confirmar essa relação de antecedência entre essas variáveis e a produção industrial, o passo seguinte é testar correlaçóes cruzadas dois a dois. Aquelas que apresentam alta correlação com a produção industrial no mesmo momento temporal são eliminadas, já que tal resultado caracteriza a existência de coincidência, ${ }^{20}$ e não de antecedência, como se deseja. Após esses dois procedimentos, podemos inferir que apenas quatro séries apresentam relaçóes antecedentes com a produção industrial; essas são mostradas na Tabela 4.
Nesse procedimento, usa-se como regra que uma antecedência de 0 a 3 meses é de curto prazo, de 4 a 6 meses de médio prazo, e de mais de 12 meses considera-se longo prazo. ${ }^{21}$ Dessa forma, podemos ver que duas variáveis contribuem para anteceder o comportamento da produção industrial no curto prazo: o pessoal ocupado na indústria de São Paulo e a taxa de juros selic. Essas, juntamente com a produção industrial nacional do segmento de metalurgia dos náo ferrosos e a venda de ônibus, também são importantes no médio prazo.

\author{
${ }^{20}$ As correlações cruzadas \\ são feitas com uma diferença \\ de seis meses, tanto para lag \\ quanto para lead. \\ ${ }^{21}$ A definição de um período \\ de 12 meses como sendo \\ longo prazo foi arbitrária. \\ O teste de causalidade, \\ porém, foi aplicado para \\ 24 lags, e os resultados em \\ pouco se modificaram.
}




\section{Gráfico 4.3 P.0. na indústria}

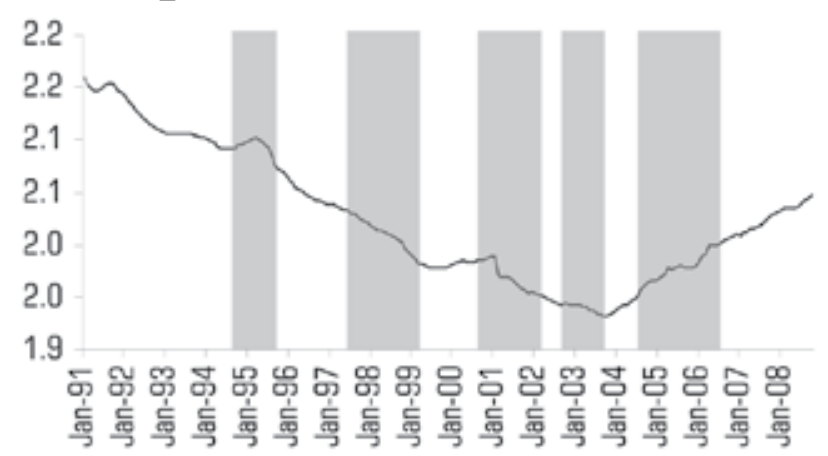

\section{Gráfico 4.4_Taxa de juros selic}

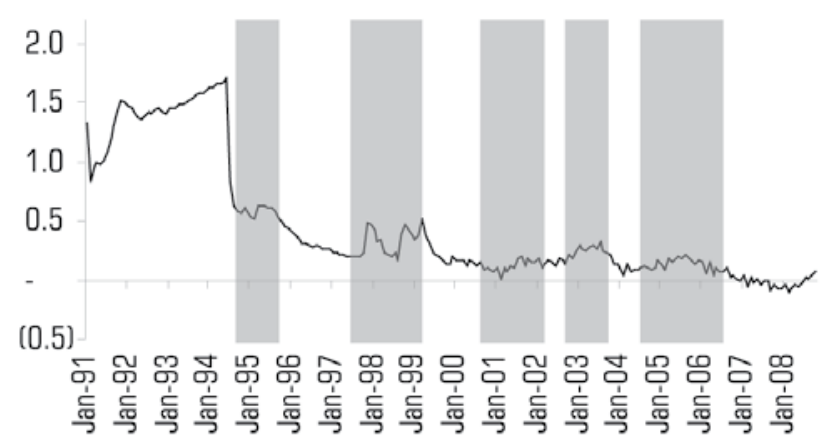

Nota: P.O.: Pessoal Ocupado.

Por fim, apenas duas são indicativas de uma antecedência de longo prazo, ou seja, mais de 12 meses, que é a venda nacional de ônibus e a taxa de juros selic. O conjunto de gráficos 4.1 a 4.4 mostra o comportamento dessas variáveis. As barras cinza sinalizam os períodos recessivos classificados para a produção industrial do Rio Grande do Sul na seção anterior.

\section{5_Conclusões}

A análise estatística dos ciclos econômicos e de indicadores antecedentes são ferramentas úteis para caracterizar períodos recessivos e de crescimento, ajudando no planejamento de governos e empresas. Valendo-se de dados da produção industrial do Rio Grande do Sul, foram testadas e comparadas três diferentes técnicas de estimativas do comportamento cíclico do setor no Estado. Com dados trimestrais de 1991-I a 2008-IV, foram encontrados cinco ciclos recessivos que permitem associar esse referente ao Estado ao comportamento da economia brasileira e também a variáveis macroeconômicas.

Nesta análise, destaque para a influência da taxa de câmbio, das elevadas taxas de juros e da queda da renda mundial. Em particular, a intempérie climática ocorrida entre 2004 e 2005 impactou a economia do Estado e contribuiu para gerar um comportamento cíclico recessivo local que foi distinto do cenário nacional.

A estimativa paramétrica permite inferir que, em média, um período recessivo dura 2,6 trimestres na indústria gaúcha, com taxa média de 1,36\% ao trimestre. Esse resultado é contrastado com 2,1 trimestres para períodos de crescimento e que apresentam taxa média trimestral de 3,2\%. 
Como forma de complementar o estudo de ciclos e permitir que esses possam ser antecipados, foram identificados os indicadores antecedentes. Partindo da análise de um conjunto de mais de 200 séries de dados econômicos regionais e nacionais e aplicando diversas ferramentas estatísticas, chega-se a quatro séries que são candidatas a antecipar o comportamento da produção industrial no Rio Grande do Sul. São elas: a produção nacional de minerais não metálicos, a venda nacional de ônibus, o emprego industrial em São Paulo e a taxa Selic. Todas com poder de previsão de curto, médio ou, então, longo prazos.

Apesar de os resultados encontrados serem úteis para o debate sobre os ciclos econômicos aplicados ao Brasil e o processo de seleção de indicadores antecedentes, a aplicação de diferentes modelos, como o probit, e a mudança de regime no formato espaço de estado ficam como sugestão de pesquisa futura. 


\section{Referências bibliográficas}

\author{
ANDREWS, D. W. K.; \\ PLOBERGER, W. Optimal tests \\ when a nuisance parameter is \\ present only under the alternative, \\ Econometrica, p. 1383-1414, 1994. \\ ARTIS, M. J.; ZHANG, W. \\ International business cycles and \\ the ERM: Is there a European \\ business cycle? International \\ Journal of Finance \& Economics, \\ vol. 2, n. 1, p. 1-16, 1997.
}

BEAULIEU, J. J.; MIRON, J. A. Seasonal unit roots in aggregate US data. Journal of Econometrics, v. 55, p. 305-328, 1993.

BRY, G.; BOSCHAN, C. Cyclical analysis of time series: Selected procedures and computer programs. NBER Technical Paper 20, 1971.

BURNS, A.; MITCHELL, W. Measuring business cycles. New York: National Bureau of Economic Research, 1946.

CANOVA, F.; CICCARELLI, M.; ORTEGA, E. Similarities and convergences in G-7 cycles. Journal of Monetary Economics, 54, p. 85-878, 2005.
CÉSPEDES, B. J. V.; CHAUVET, M.; LIMA, E. C. R. Forecasting Brazilian output and its turning points in the presence of breaks: A comparison of linear and nonlinear models. Estudos Econômicos, v. 36, n. 1, p. 5-46, Mar. 2006.

CHAUVET, M. An econometric characterization of business cycle dynamics with factor structure and regime switching, International Economic Review, vol. 39, n. 4, p. 969-96, 1998.

CHAUVET, M.; MORAIS, I. A. C. Predicting recessions in Brazil, presented in 2008 annual meeting LAMES/LACEA. Rio de Janeiro, Brasil, 2008.

DUARTE, A. J. M.; ISSLER, J. $\mathrm{V}$.; SPACOV, A. Indicadores coincidentes de atividade econômica e uma cronologia de recessōes para o Brasil, Pesquisa e Planejamento Econômico. PPE, v. 34, n. 1, p. 1-38, 2004.

ENGEL, C.; HAMILTON, J. D. Long swings in the dollar: Are they in the data and do markets know it? American Economic Review, 80, p. 689-713, 1990.
ENGLE, R. F.; ISSLER, J. V. Common trends and common cycles in Latin America RBE, vol. 47, n. 2, p. 149-76, 1993.

ESTRELLA, A.; MISHKIN, F. S. The predictive power of the term structure of interest rates in Europe and the United States: Implications for the European Central Banks. European Economic Review, vol. 41, n. 7, p. 1357-1401, 1998.

GARCIA, R. Asymptotic null distribution of the likelihood ratio test in Markov switching models. International Economic Review, vol. 39, n. 3, p. 763-788, 1998.

GREGORY, A. W.; HEAD, A. C.; RAYNAULD, J. Measuring world business cycles. International Economic Review, vol. 38, p. 677-702, 1997.

HAMILTON, J. D. A new approach to the economic analysis of nonstationary time series and the business cycle.

Econometrica, vol. 57 ,

p. 357-384, 1989.
HAMILTON, J. D. Analysis of time series subject to changes in regime. Journal of Econometrics, v. 45, p. 39-70, 1990.

HAMILTON, J. D. A quasi-

Bayesian approach to estimating parameters for mixtures of normal distributions. Journal of Business and Economic Statistics, 9, p. 27-39, 1991.

HAMILTON, J. D. Specification testing in Markov-Switching time series models. Journal of Econometrics, 70, p. 127-157, 1996.

HANSEN, B. E. The likelihood ratio test under non-standard conditions: Testing the Markov switching model of GNP. Journal of Applied Econometrics, 7 , p. S61-S82, 1992.

HARDING, D.; PAGAN, A. A comparison of two business cycle dating methods. Journal of Economic Dynamics and Control, vol. 27, n. 9, p. 1681-1690, 2003.

HARDING, D.; PAGAN, A. A suggested framework for classifying the modes of cycle research. Journal of Applied Econometrics, 20, p. 151-159, 2005. 
HYLLEBERG, S.; ENGLE, R. F.; GRANGER, C. W. J.; YOO, B.S. Seasonal integration and cointegration. Journal of Econometrics, 44, p. 215-238, 1990.

\section{IBRE - COMITÊ DE DATAÇĀO} DE CICLOS ECONÔMICOS, Fundação Getúlio Vargas, Instituto Brasileiro de Economia, Rio de Janeiro, 27 de maio de 2009

ISSLER, J. V.; VAHID, F. The missing link: Using the NBER recession indicator to construct coincident and leading indices of economic activity. Journal of Econometrics, vol. 132, n. 1, p. 281-303, 2006.

KIM, C-J; NELSON, C. R. State-Space models with regime switching - Classical and GibbsSampling approaches with applications. MIT Press, 2nd ed., 2000.

KOSE, M. A.; OTROK, C.; WHITEMAN, C. H. International business cycles: World, region, and country-specific factor., American Economic Review, vol. 93, n. 4, p. 1216-1239, 2003.

KOSE, M. A.; OTROK, C.; PRASAD, E. S. Global business cycles: Convergence or decoupling? NBER Working Paper $\mathrm{n}^{\circ}$ 14292, 2008.

LEE, J.; STRAZICICH, M. C. Minimum lm unit root test with one structural break. Appalachian State University Working Paper, 2004.
MORAIS, I. A. C. 2005: Um ano difícil para a indústria do Rio Grande do Sul. In: SANTI, Maria Fernanda Cavalieri de Lima (Org.). Conjuntura política e econômica do Rio Grande do Sul: Uma análise da década de 2000, 1.ed.. Porto Alegre, Nova Prova, v. 1, p. 65-77, 2006.

MORAIS, I. A. C.; FRAINER, V. Uma investigação sobre o ciclo dos negócios na indústria da Bahia. Revista Econômica do Nordeste, Fortaleza, v. 36, n. 3, p. 378-410, 2005.

MORAIS, I. A. C.; FRAINER, V. Divergência e distribuição industrial no Rio Grande do Sul (1985-2003). Análise Econômica (UFRGS), n. 49, p. 211-232, 2008.

MORAIS, I. A. C.; PORTUGAL, M. S. Um novo índice coincidente para a atividade industrial do Estado do Rio Grande do Sul. Estudos Econômicos, 37, p.35-70, 2007.

MORAIS, I. A. C.; PORTUGAL. M. $S$. Business cycle in the industrial production of Brazilian States. Análise Econômica (UFRGS), v. 50, p. 1-20, 2008.

MORGAN, M. S. The history of econometric ideas - Historical perspectives on modern economics. Cambridge University Press, Cambridge, 1990.

NILSSON, R.; GUIDETTI, E. Predicting the business cycle: How good are early estimates of OECD composite leading indicators? OECD Statistics Brief $\mathrm{n}^{\circ} 14,2008$.
OECD. OECD system of composite leading indicator, Disponível em: <http://www.oecd.org/ dataoecd/26/39/41629509.pdf.>.

PICCHETTI, P.; TOLEDO, C. Estimating and interpreting a common stochastic component for the Brazilian industrial production index. $R B E$, vol. 56, n. 1, p. 107-120, 2002.

SEIP, K. L.; McNOWN, R. The timing and accuracy of leading and lagging business cycle indicators: A new approach. International Journal of Forecasting, 23, p. 277-287, 2007.

RUUD, P. A. Extension of estimation methods using the EM-algorithm, Journal of Econometrics, 49, 305-341, 1991.

STOCK, J. H.; WATSON, M. W.

A new approach to leading economic indicators. Working Paper: Harvard University, Kennedy School of Government, 1988.

STOCK, J. H.; WATSON, M. W. New indexes of leading and coincident economic indicators. NBER Macroeconomic Annual, p. 351-95, 1989.

STOCK, J. H.; WATSON, M. W. A probability model of the coincident economic indicators. In: LAHIRI, K.; Moore, G. (Eds.). Leading Economic Indicators: New Approaches and Forecasting Records, New York: Cambridge University Press, 1991.
STOCK, J. H.; WATSON, M. W. A procedure for predicting recessions with leading economic indicators: Econometric issues and recent experience. In: STOCK, J. H.; WATSON, M. W. (Eds.). New research on Business Cycles, Indicators and Forecasting, Chicago: University of Chicago Press, for NBER, p. 255-284, 1993.

STOCK, J. H.; WATSON, M. W. Understanding changes in International Business Cycle Dynamics. Journal of the European Economic Association, vol. 3, n. 5, p. 969-1006, 2005.

ZARNOWITZ, V. Business cycles: Theory, history, indicators, and forecasting. The University of Chicago Press, Chicago, 1996.

ZIVOT, E.; ANDREWS, D. W. K. Further evidence on the great crash, the oil-price shock and the unit root hypothesis. Journal of Business and Economic Statistics, vol. n. 10, p. 251-270, 1992.

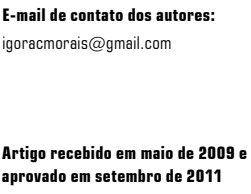


Anexo 1

M(2)-AR(p) - Produção industrial do Rio Grande do Su

\begin{tabular}{|c|c|c|c|c|}
\hline & MSM(2)-AR(1) & MSM(2)-AR(2) & MSM(2)-AR(3) & MSM(2)-AR(4) \\
\hline$\mu_{1}$ & $-1,68(0,93)$ & $-1,17(0,20)$ & $-1,23(0,23)$ & $-1,36(0,20)$ \\
\hline$\mu_{2}$ & $2,26(0,71)$ & $3,16(0,30)$ & $3,24(0,28)$ & $3,24(0,25)$ \\
\hline$\varphi_{1}$ & $-0,06(0,33)$ & $-0,37(0,08)$ & $-0,23(0,08)$ & $-0,07(0,07)$ \\
\hline$\varphi_{2}$ & & $-0,48(0,07)$ & $-0,51(0,07)$ & $-0,61(0,07)$ \\
\hline$\varphi_{3}$ & & & $0,13(0,07)$ & $0,25(0,06)$ \\
\hline$\varphi_{4}$ & & & & $-0,15(0,05)$ \\
\hline$\sigma$ & 1,86 & 1,36 & 1,30 & 1,21 \\
\hline$p_{1}$ & 0,38 & 0,55 & 0,56 & 0,55 \\
\hline Duração & 2,12 & 3,08 & 2,87 & 2,63 \\
\hline$p_{2}$ & 0,61 & 0,44 & 0,43 & 0,44 \\
\hline Duração & 3,35 & 2,43 & 2,24 & 2,17 \\
\hline$p_{11}$ & 0,52 & 0,67 & 0,65 & 0,62 \\
\hline$p_{22}$ & 0,70 & 0,58 & 0,55 & 0,53 \\
\hline AIC & 5,01 & 4,93 & 4,94 & 4,91 \\
\hline $\mathrm{HQ}$. & 5,09 & 5,03 & 5,05 & 5,04 \\
\hline
\end{tabular}


Anexo 2
Séries antecedentes da produção industrial do Rio Grande do Sul Selecionadas pelo teste de causalidade de Granger

\begin{tabular}{l|r|r} 
Série & lags & Fonte \\
\hline Extraçáo de minerais náo metálicos & 3 & IBGE \\
\hline Artefatos diversos de borracha & 3 & IBGE \\
\hline Laminados de material plástico & 3 & IBGE \\
\hline Laminados, relaminados e trefilados de aço & 3 & IB GE \\
\hline Metalurgia dos não ferrosos & 6 & IBGE \\
\hline Máquinas e equipamentos para fins industriais e comerciais & 6 & IBGE \\
\hline Caminhóes e ônibus, inclusive motores & 6 & IBGE \\
\hline Horas trabalhadas - na produção - ind. - índice (média 2006 100) - SP & 6 & Fiesp \\
\hline Horas pagas - indústria - índice (média 2006 =100) - SP & 6 & Fiesp \\
\hline Material de embalagem de papel, papeláo e cartáo & 3,6 e 12 & IBGE \\
\hline Artefatos de concreto, cimento e fibrocimento & 3,6 e 12 & IBGE \\
\hline Embalagens metálicas & 6 e 12 & IBGE \\
\hline Consumo aparente - gás GLP - média - qde./dia - bbrril (mil) & 6 e 12 & ANP \\
\hline Vendas - ônibus - nacionais - Unidade & 6 e 12 & Anfavea \\
\hline Pessoal ocupado - indústria - índice (média 2006 = 100) - SP & 3 e 6 & Fiesp \\
\hline Expedição de caixas, acess. e chapas - papelão ondulado - tonelada & 3,6 e 12 & ABPO \\
\hline Taxa de juros - Over / Selic - (\% a.m.) & BCB \\
\hline Consumo aparente - derivados de petróleo - média - qde./dia - Barril (mil) & 12 & ANP \\
\hline
\end{tabular}

